\title{
ON-CHIP PHASE SHIFT REFRACTOMETER VIA MICROFLUIDIC LIQUID GRATING
}

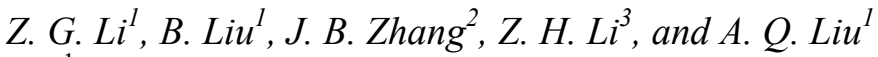 \\ ${ }^{1}$ School of Electrical and Electronic Engineering \\ Nanyang Technological University, Singapore 639798 \\ ${ }^{2}$ Data Storage Institute, 5 Engineering Drive 1, Singapore 117608 \\ ${ }^{3}$ Institute of Mechanics, Chinese Academy of Sciences, Beijing100190, PR China
}

\begin{abstract}
This paper describes the design, realization and testing of a refractometer based on the principle of transmission phase grating. An integrated multichannel microfluidic chip is used as a basic optical component for building the refractometer. A laser light beam is coupled to the top of the microfluidic chip and the generated diffraction pattern is recorded by a CCD camera and the intensities of the $0^{\text {th }}$ and $\pm 1^{\text {st }}$ order are further analyzed. The microfluidic chip is fabricated using standard soft lithography technology. The realized refractometer has a refractive index resolution of $1.0 \times 10^{-5}$.
\end{abstract}

\section{KEYWORDS}

Refractometer, transmission phase shift grating, multichannel mircofluidic chip

\section{INTRODUCTION}

The integration of microfluidic and optical components into a single miniaturized platform is one of the rapidly growing areas of applied science and technology with numerous applications in biology, chemistry, and biochemistry [1-4]. Most often, microfluidic optical components can be constructed through microchannels themselves or can be adjusted by changing the shapes of the microchannels and refractive indices of liquids in them [5-8]. In additional to the integration of the microfluidic and optical components, adaptive optical devices made using the microfluidic technology can also be used as stand-alone optical elements.

Among all, optical grating is one of the optical components which can be realized through the construction of multichannels in a mirofluidic chip. Compared to the traditional solid gratings, microfluidic liquid gratings provide more tuning possibilities and are different compared to other liquid crystal materials [9] and polymers [10]. Liquid tunable gratings can be developed in various devices such as optical switch, wavelength splitter, refractometer and etc [11].

In this paper, we present a refractometer based on the principle of transmission phase grating and demonstrate its usefulness in sensing applications. Section 2 describes the design and fabrication of the microfluidic chip. Section 3 illustrates the principle of transmission phase grating. Section 4 shows the experimental results. Section 5 concludes.

\section{DESIGN AND FABRICATION}

The schematic of the refractometer based on liquid tunable transmission phase grating is illustrated in Fig. 1. It consists of a single mode optical fiber, a collimating lens, a microfluidic chip, a collecting lens and a CCD camera. The single mode optical fiber and collimating lens are used to couple input laser light beam to the liquid transmission grating, and the output beams are overlapped in order to generate a diffraction pattern collected by the collecting lens and detected by the CCD camera. When different types of biological or chemical solutions with different refractive indices are injected by syringe pumps into the multichannels of the microfluidic chip, the output diffraction patterns shift accordingly. In this paper, the wavelength of the input laser light beam $\lambda$ is $632.8 \mathrm{~nm}$ (He-Ne semiconductor laser).

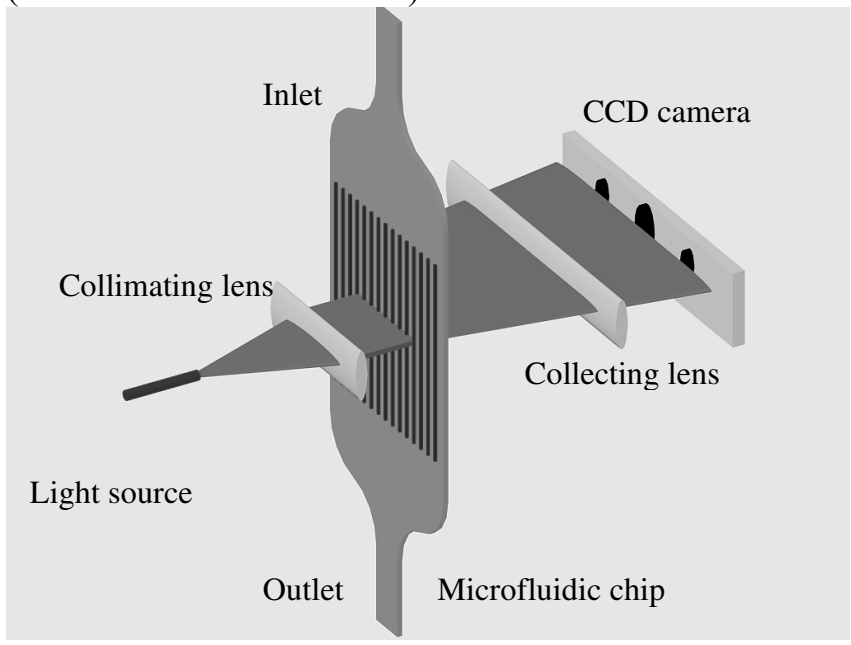

Figure 1: Schematic of the refractometer based on liquid tunable transmission phase grating including light source, collimating lens, microfluidic chip, collecting lens and CCD camera.

The top view and cross section of the microfluidic chip is shown in Fig. 2(a) and 2(b), respectively. The microfluidic chip is fabricated by polydimethyl siloxane (PDMS) with soft lithography technology. The liquid samples in the microchannels and the PDMS structures form an alternative grating structure. In this paper, the grating pitch $\Lambda$ is $40 \mu \mathrm{m}$ and the thickness of the microchannel $t$ is 32.3 $\mu \mathrm{m}$. 


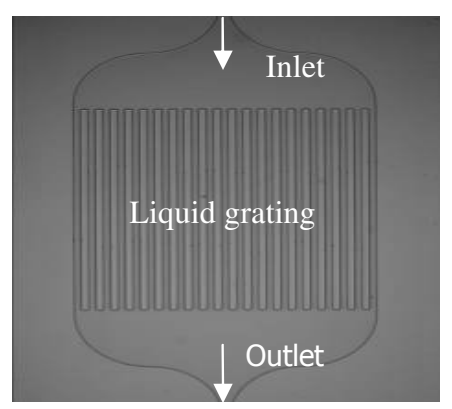

(a)

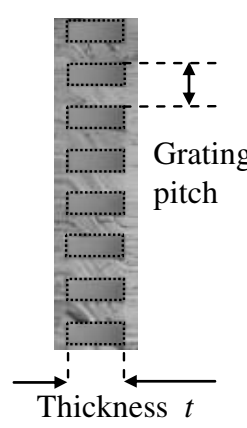

(b)
Figure 2: Microfluidic chip with liquid grating, (a) top view and (b) cross-sectional view.

\section{TRANSMISSION PHASE GRATING}

The principle of the refractometer is based on the generation of the diffraction pattern as a result of the overlap of coherent light beams from a transmission phase grating, which consists of $2 \mathrm{~N}$ alternate contiguous regions in a transparent medium as depicted in Fig. 2.

In the transmission phase grating, the intensity of the $0^{\text {th }}$ order of the diffracted irradiance pattern $[12,13] I_{\mathrm{m}=0}$ can be expressed as:

$$
I_{m=0}=I_{0} \cos ^{2} \frac{\pi t}{\lambda} \Delta n, \quad \Delta n=n_{1}-n_{2}
$$

where $t$ is the thickness of the microchannel, $\lambda$ is the wavelength of the input light beam, $I_{0}$ is the maximum intensity of the $0^{\text {th }}$ order of the diffraction patterns, which can be normalized to 1 , and $n_{1}$ and $n_{2}$ are the refractive indices of two different transparent mediums, respectively. Based on Eq. (1), the contrast of the refractive indices between two transparent regions can be derived as:

$$
\Delta n=\frac{\lambda}{\pi \cdot t} \cdot\left[\arccos \left(\left(I_{m=0}\right)^{\frac{1}{2}}\right)\right]
$$

Hence, the contrast refractive index can be determined by measuring the intensity shift of the diffraction pattern. For a given resolution of the CCD camera and a fixed light source, the sensitivity of the device towards the refractive index change is proportional to the thickness of the microchannel.

\section{EXPERIMENT RESULTS}

The diffraction patterns of different volume ratios of aqueous isopropanol (IPA) solutions are measured as shown in Fig. 3. The diffraction pattern is tuned by shifting the refractive index of the liquid samples. The change in the refractive index of the IPA solutions (the volume ratio of the IPA from $82 \%$ to $98 \%$ ), alters the intensities of the $0^{\text {th }}$ and $\pm 1^{\text {st }}$ order accordingly.

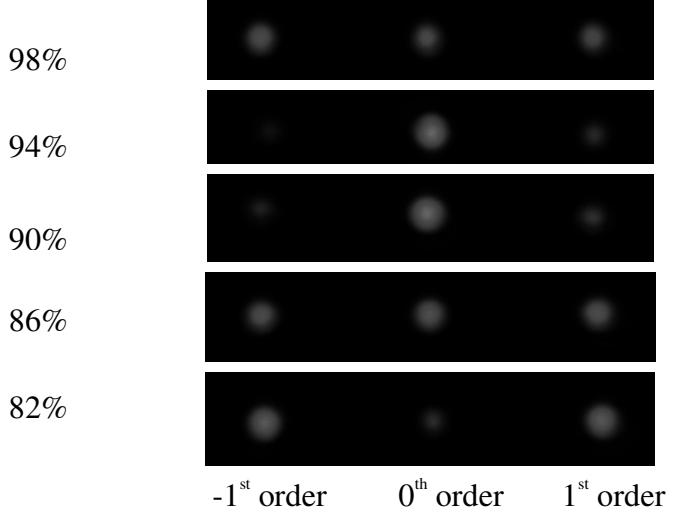

Figure 3: Different diffraction intensity pattern vs. different volume ratios of IPA solutions.

Figure 4 depicts the measured relations between the normalized intensity of the $0^{\text {th }}$ order diffraction pattern (Fig. 3) and the refractive index contrast between different IPA solution and PDMS. The normalized $0^{\text {th }}$ order intensity will shift periodically and achieve the maximum when the phase shift is 0 or $2 m \pi(m=1,2 \ldots)$.

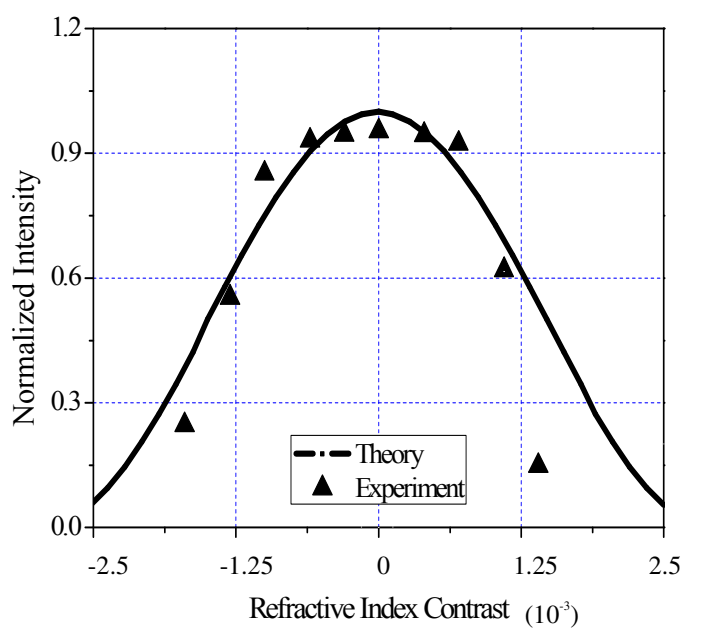

Figure 4: Refractive index contrast vs. normalized intensity. The liquid samples are different volume ratio IPA solutions, from $80 \%$ to $98 \%$.

Furthermore, Fig. 5 shows the variation of the ratio of the intensities of the $0^{\text {th }}$ order to the $1^{\text {st }}$ order with the refractive index contrast from $-1.25 \times 10^{-3}$ to $1.25 \times 10^{-3}$. Table 1 shows the measured results of three different types of IPA solutions. The measured results agree well with the theoretical values. 


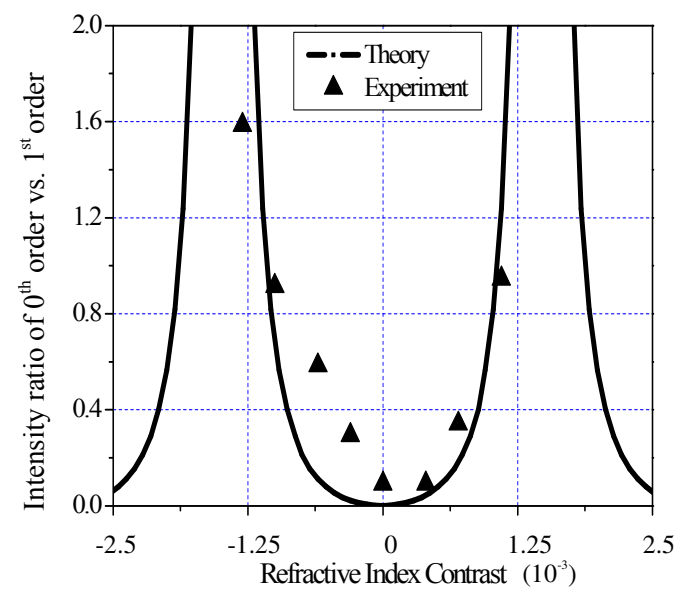

Figure 5: The ratio of intensity vs. the refractive index contrast.

Table 1: Refractive index measurement of IPA solutions

\begin{tabular}{|l|c|c|c|}
\hline Sample & $\begin{array}{c}\text { RI } \\
\text { (theory) }\end{array}$ & $\begin{array}{c}\text { RI } \\
\text { (measured) }\end{array}$ & $\begin{array}{c}\text { Volume ratio } \\
\text { (\%) }\end{array}$ \\
\hline IPA1 & 1.37658 & 1.37668 & 94 \\
\hline IPA2 & 1.37556 & 1.37544 & 88 \\
\hline IPA3 & 1.37488 & 1.37448 & 84 \\
\hline
\end{tabular}

\section{CONCLUSIONS}

In conclusion, a refractometer based on the principle of transmission phase grating is reported. An integrated multichannel microfluidic chip is used as the basic optical component for constructing the refractometer. The generated diffraction pattern is recorded by a CCD camera and the intensities of the $0^{\text {th }}$ and $\pm 1^{\text {st }}$ order are further analyzed. The microfluidic chip is fabricated in standard soft lithography technology. The realized refractometer has a refractive index resolution of $1.0 \times 10^{-5}$. This on-chip refractometer developed is foreseen to have broad potential applications in the refractive index measurement for biological and chemical analysis.

\section{REFERENCES}

[1] G. M. Whitesides, "The origins and the future of microfluidics," Nature, vol. 442, pp. 368-373, 2006

[2] G. H. W. Sanders and A. Manz, "Chip-based microsystems for genomic and proteomic analysis," TrAC-Trends Anal. Chem., vol. 19, pp. 364-378, 2000
[3] C. Monat, P. Domachuk, and B. J. Eggleton, "Integrated optofluidics: A new river of light," Nat. Photonics., vol. 1, pp. 106-114, 2007

[4] U. Levy and R. Shamai, "Tunable optofluidic devices," Microfluid. Nanofluid., vol. 4, pp. 97-105, 2008.

[5] N. Chronis, G. L. Liu, K. H. Jeong, and L. P. Lee, "Tunable liquid-filled microlens array integrated with microfluidic network," Opt. Express., vol. 11, pp. 2370-2378, 2003.

[6] D. B. Wolfe, D. V. Vezenov, B. T. Mayers, G. M. Whitesides, R. S. Conroy, and M. G. Prentiss, "Diffusioncontrolled optical elements for optofluidics," Appl. Phys. Lett., vol. 87, 181105, 2005.

[7] L. K. Chin, A. Q. Liu, J. B. Zhang, C. S. Lim, and Y. C. Soh, "An on-chip liquid tunable grating using multiphase droplet microfluidics", Appl. Phys. Lett., vol. 93, 164107, 2008

[8] Y. C. Seow, A. Q. Liu, L. K. Chin, X. C. Li, H. J. Huang, T. H. Cheng, and X. Q. Zhou, "Different curvatures of tunable liquid microlens via the control of laminar flow rate", Appl. Phys. Lett., vol. 93, 084101, 2008

[9] S. W. Ke, T. H. Lin and A. Y. Fuh, "Tunable Grating Based on Stressed Liquid Crystal", Opt. Express., vol. 16, pp. 2062-2067, 2008.

[10] J. Shi, V. K. S. Hsiao and T. J. Huang, "Nanoporous Polymeric Transmission Gratings for High-speed Humidity Sensing", Nanotechnology., vol. 18, 465501, 2007

[11] A. Groisman, S. Zamek, K. Campbel, L. Pang, U. Levy and Y. Fainman, "Optofluidic $1 \times 4$ Switch", Opt. Express., vol. 18, pp. 13499-13508, 2008.

[12] J. A. Brandao Faria, "A Transmission phase grating analysis", Microwave Opt Technol Lett., vol. 4, pp. 224-227, 1991

[13] E. Hecht, Optics, Addison-Wesley, Reading, Massachusetts, 2002.

\section{CONTACT}

* A.Q. Liu, tel: +65-6790-4336; eaqliu@ntu.edu.sg; URL: http://nocweba.ntu.edu.sg/laq_mems/ 